\title{
Medios semióticos de objetivación y procesos de objetivación en estudiantes de sexto grado cuando resuelven tareas de tipo multiplicativo ${ }^{1}$
}

\section{Semiotic means of objectification and objectification processes of sixth grade students when solving tasks multiplicative.}

Meios semióticos de processos de estudantes da sexta série objetivação e coisificação quando resolver tarefas multiplicativo.

\section{Resumen}

Se presenta la hipótesis de investigación de la tesis de maestría "Procesos de objetivación y medios semióticos de objetivación en estudiantes de sexto grado cuando resuelven tareas de tipo multiplicativo" que se viene desarrollando en la Maestría en Educación con énfasis en educación matemática de la Universidad Distrital Francisco José de Caldas. El estudio se sitúa en la perspectiva sociocultural del aprendizaje de las matemáticas y utiliza los constructos de la teoría cultural de la objetivación para analizar las acciones de los estudiantes e interpretar sus producciones cuando resuelven tareas de tipo multiplicativo, toda vez que se desea estudiar los medios semióticos de objetivación y procesos de objetivación emergentes en los estudiantes cuando resuelven este tipo de tareas.

Palabras clave: Procesos de objetivación; medios semióticos de objetivación; matemáticas escolares; operaciones aritméticas; multiplicación.

\begin{abstract}
It presents the research hypothesis master thesis "objectification processes and semiotic means of objectification in sixth grade students when solving tasks multiplicative" that is being developed in the Master of Education with emphasis in mathematics education at the University District Francisco José de Caldas. The studio is located in the sociocultural perspective of learning mathematics and uses the constructs of cultural objectification theory to analyze the actions of students and interpret their productions when solving tasks multiplicative, since you want to explore ways semiotic objectification and objectification processes emerging in students when solving these tasks.
\end{abstract}

Keywords: Process objectification semiotic means of objectification school mathematics, arithmetic, multiplication.

1 Artíulo de Investigación

2 Universidad Distrital Francisco José de Caldas. Contacto: javiermojicav@hotmail.com 


\section{Resumo}

Apresenta-se a hipótese de Dissertação de Mestrado "processos de objetivação e meios semióticos de objetificação em estudantes da sexta série na resolução de tarefas multiplicativo" de pesquisa que está sendo desenvolvida no Mestrado em Educação, com ênfase em educação matemática na Universidade Distrital Francisco José de Caldas. O estúdio está localizado na perspectiva sociocultural da aprendizagem da matemática e usa as construções da teoria da objetivação cultural para analisar as ações dos alunos e interpretar as suas produções ao resolver tarefas multiplicativo, desde que você queira explorar formas objetivação semiótica e processos de objetivação emergentes em estudantes ao resolver essas tarefas.

Palavras-chave: Processo de objetivação meios semiótica da matemática escolar objetivação, a aritmética, a multiplicação.

\section{Presentación del problema}

Parte de la investigación en didáctica de las matemáticas hace posible distinguir en el tiempo una tendencia que durante algunas décadas estuvo orientada a describir las dificultades presentadas por los estudiantes cuando tienen contacto con algunos objetos matemáticos, esta información, que si bien, aporta a la comprensión de diversas situaciones del aula al parecer no ha logrado transformar radicalmente algunas maneras y procederes en la enseñanza de las matemáticas. Este hecho, acompañado de una conciencia colectiva que concibe el aula como un complejo entramado de relaciones, imaginarios, saberes, intenciones y sujetos concretos hace que las investigaciones en didáctica de las matemáticas empiecen a centrarse en otros fenómenos que generan a su vez nuevos paradigmas de investigación (Radford, 2011). Las aproximaciones socioculturales consideran el aula y los fenómenos de enseñanza y aprendizaje como dinámicos y emergentes. El estudio de estos fenómenos puede llegar a dar cuenta de diversos aspectos del aprendizaje de las matemáticas, de cómo piensan los estudiantes, la anatomía de sus razonamientos, el reconocimiento de sus potencialidades, de aquello que pueden hacer los estudiantes con lo que saben, del tipo de acercamiento a saberes construidos histórico-culturalmente y las maneras de comprender por qué hacen lo que hacen. De tal suerte que desde los hallazgos de estas investigaciones puedan generarse estrategias de enseñanza que teniendo en cuenta las maneras de aprender de los escolares, apunten a transformar las prácticas de la enseñanza y el aprendizaje de las matemáticas.

El complejo ambiente del aula esconde en su interior un sin número de expresiones dadas por los estudiantes cuando aprenden, que a veces nos parecen naturales, pero no nos detenemos a pensar en la información que ellas nos pueden ofrecer; centrarnos en su análisis ofrece la posibilidad, poco explotada, de tomar conciencia de estos signos como hechos latentes de comprensión que pueden llegar a transformar, por ejemplo, la manera como juzgamos o "evaluamos" las comprensiones o aprendizajes de los estudiantes. Por ello, el problema está en reconocer estos signos o medios semióticos de objetivación de manera que permita traducir estas acciones en orientaciones para la enseñanza. En términos de Radford (2013) los seres humanos llevan a cabo operaciones a través de signos que modifican de manera fundamental la manera en que llegamos a pensar y conocer.

En el estudio que se está desarrollando se espera analizar los medios semióticos de objetivación y procesos de objetivación desarrollados por estudiantes de grado sexto en un colegio público de la ciudad de Bogotá - Colombia cuando resuelven tareas de tipo multiplicativo utilizando los constructos de la teoría cultural de la objetivación, pues aunque se han generado abundantes pronunciamientos frente a dificultades y posibilidades en el campo multiplicativo aún no se han explorado cuales serían los medios semióticos de objetivación movilizados por los estudiantes cuando afrontan 
este tipo de tareas, de manera que esta información permita comprender las formas de reflexión de los estudiantes frente al objeto cultural de la multiplicación.

\section{Marco de referencia conceptual}

Como se mencionó nos posicionamos desde una postura que comprende el aprendizaje y la enseñanza desde una perspectiva semiótico cultural de la educación matemática, en la cual acudimos a la teoría cultural de la objetivación la cual "aboga por una concepción no mentalista del pensamiento y por una idea de aprendizaje tematizado como adquisición comunitaria de formas de reflexión del mundo guiadas por modos epistémico-culturales históricamente formados." (Radford, 2006, p. 105). Pragmáticamente la teoría sugiere prestar atención a los medios semióticos de objetivación que utiliza el alumno cuando resuelve tareas matemáticas, en un esfuerzo que es, a la vez, elaboración de significados y toma de conciencia de los objetos conceptuales.

Los medios semióticos de objetivación son entendidos como los objetos, herramientas, recursos lingüísticos y signos que las personas intencionalmente usan en la construcción social de significados con el fin de lograr una forma estable de conciencia, hacer evidentes sus intenciones, yllevar a cabo un despliegue de acciones para alcanzar el objetivo de sus actividades (Radford, 2008, 2010). Entre estos signos observables en la conducta del sujeto se hallan los símbolos, los gestos, los movimientos, las señas, el lenguaje y las interacciones; los cuales permiten dar cuenta de una intención comunicativa, una manifestación que pone algo de presente, toda vez que "se convierten en constituyentes mismos del acto cognitivo que posiciona al objeto conceptual no dentro de la cabeza sino en el plano social" (Radford, 2006, p.125), además estratifican el objeto matemático en estratos de generalidad de acuerdo con la actividad reflexiva que ellos median. En resumen, los medios semióticos de objetivación corresponden a "los objetos y signos utilizados para objetivar el conocimiento" (Vergel, 2012, p. 21)
El aprendizaje no consiste en construir o reconstruir un conocimiento sino que "se trata de dotar de sentido a los objetos conceptuales que encuentra el alumno en su cultura" (Radford, 2006, p. 113) a través de un proceso social, sensible y material de objetivación en el cual "el alumno alcanza una comprensión crítica, a través de dotación de significados, de los objetos culturales matemáticos y de la lógica cultural de éstos." (Radford, 2011, p. 45) en donde tiene lugar la participación y la internalización, que permiten el paso de lo social a lo individual.

\section{Acerca de las tareas de tipo multiplicativo}

Cuando nos referimos a tareas de tipo multiplicativo estamos refiriendo tareas que para su solución requieren el uso de la multiplicación o la división, que aunque podría relacionarse con las situaciones $^{3}$ de estructura multiplicativa del campo conceptual multiplicativo de Vergnaud (1994), no corresponden fielmente a dicho enfoque en tanto los postulados epistemológicos de Verganud discrepan de los adoptados por Radford, por el contrario, el enfoque asumido nos lleva a considerar los planteamientos de La Teoría de la Actividad de Leontiev (1977) para el cual una actividad es considerada como

un proceso social cuyo propósito es alcanzar un objetivo impregnado de entrada con significados culturales y conceptuales, objetivo que se alcanza a través de acciones mediatizadas por sistemas semióticos depositarios de la historia cognitiva escrita en estos últimos por generaciones pasadas (Radford, 2008, p. 741).

\section{Metodología y análisis de datos}

El estudio asume un enfoque de investigación cualitativa de tipo descriptivo e interpretativo (Latorre, Del Rincón y Arnal, 2003). Particularmente se sigue el modelo de análisis multimodal usado por Miranda, Radford y Guzmán (2007) fundamentado en los planteamientos de Arzarello (2006), en el cual la información se recopila a través de video grabaciones de las cuales se seleccionan episodios y

3 "El concepto de situación no tiene aquí el sentido de situación didáctica sino más bien el de tarea, la idea es que toda situación compleja se puede analizar como una combinación de tareas de las que es importante conocer la naturaleza y la dificultad propias" 
fragmentos de la actividad matemática, a partir de los cuales se realiza el análisis multimodal, el cual debe tener en cuenta la relación de los diferentes sistemas semióticos movilizados durante la actividad (el sistema semiótico del lenguaje escrito, el del lenguaje hablado, el de los gestos, las acciones, etc.). En otras palabras, ni lo escrito, ni lo hablado, ni lo gesteado por los estudiantes es analizado de manera aislada. Antes bien, estas formas de expresión se estudian como partes clave del proceso de objetivación. (Miranda, Radford y Guzmán. 2007, p. 12)

El estudio se encuentra en una fase preliminar de pilotaje, en la cual se diseñan e implementan las tareas de tipo multiplicativo con el fin de explorar que recursos semióticos movilizan los estudiantes, depurar el tipo de preguntas propuestas y analizar el tipo de interacción que provocan. Además de la fase anterior (Fase 0) en este estudio se plantean las siguientes fases: Fase 1. Diseño de tareas. Fase 2. Implementación de las tareas y recolección de información. Fase 3. Selección de datos. Fase 4. Análisis e interpretación de datos. Fase 5. Entrevistas basadas en los hallazgos del análisis.

La población del estudio corresponde a un grupo de estudiantes de grado sexto con edades entre los 10 y 13 años del colegio Delia Zapata Olivella en la jornada tarde.

Para el análisis de los datos se cuenta inicialmente con los medios semióticos y procesos de objetivación reportados en la literatura, entre los medios semióticos encontramos señalamientos, inscripciones y ritmo, dentro de los procesos de objetivación se hallan la iconicidad y la contracción semiótica.

En un ejercicio inicial de diseño de tareas se analiza el fragmento de una actividad propuesta a una niña de 12 años al resolver una tarea de tipo multiplicativo en la que se halla evidencia que comprueba parcialmente la hipótesis que acá se sostiene.

\section{Conclusiones}

La investigación en curso espera poder generar información documentada frente a los medios semióticos de objetivación y procesos de objetivación desarrollados por estudiantes de grado sexto cuando resuelven tareas de tipo multiplicativo basados en la hipótesis inicial que considera que los constructos de la teoría cultural de la objetivación no son exclusivos del pensamiento algebraico y que pueden ser usados en otros dominios como el pensamiento multiplicativo aún cuando hasta el momento no se tengan una caracterización, ni siquiera parcial, de sus elementos constitutivos. Sin embargo, en lo explorado hasta el momento algunos ejercicios iníciales parecen demostrar la existencia de unos medios semióticos de objetivación que permiten una primera aproximación a lo semiótico de lo multiplicativo.

\section{Referencias}

Arzarello, F. (2006). Semiosis as a multimodal process. Revista Latinoamericana de Investigación en Matemática Educativa, número especial sobre semiótica, cultura y pensamiento matemático, 267-299.

Latorre. A, Del Rincón. D y Arnal, J (2003). Bases metodológicas de la investigación educativa. España. Ediciones Experiencia SL.

Miranda, I., Radford, L. y Guzmán, J. (2007). Interpretación de gráficas cartesianas sobre el movimiento desde el punto de vista de la teoría de la objetivación. Educación Matemática, 19(3), 5-30.

Radford, L. (2006). Elementos de una teoría culturaldelaobjetivación. RevistaLatinoamericana de Investigación en Matemática Educativa, número especial sobre semiótica, cultura y pensamiento matemático. 103-129.

Radford, L. (2008). Semiótica cultural y cognición. En R. Cantoral, O. Covián, R. Farfán, J. Lezama \& A. Romo (Eds.). Investigaciones sobre Enseñanza y aprendizaje de las matemáticas. Un reporte iberoamericano. (pp. 731-754). México: Diaz de Santos 
Radford, L. (2010). Layers of generality and types of generalization in pattern activities. PNA, 4(2), 37-62.

Radford, L. (2011). La evolución de paradigmas y perspectivas en la investigación. El caso de la didáctica de las matemáticas. En J. Vallès, D. Álvarez y R. Rickenmann (Eds.), L'ctivitat docent intervenció, innovació, investigación. (pp. 33-49). Girona: Documenta Universitaria

Radford, L. (2013). Three key concepts of the theory of objectification: Knowledge, knowing and learning. Journal of Research in Mathematics Education, 2(1), 7-44.

Vergel, R. (2012). Formas de pensamiento alge braico temprano en alumnos de cuarto y quinto grados de Educación Básica Primaria
(9-10 años). Proyecto doctoral. Doctorado interinstitucional en educación, énfasis en Educación Matemática. Universidad Distrital Francisco José de Caldas. Bogotá, Colombia.

Vergnaud, G. (1990). La teoría de los campos conceptuales. Recherches en Didáctique des Mathématiques, 10(2,3), 133-170.

Vergnaud, G. (1994). Multiplicative conceptual field: what and why? En: Harel, G \& Confrey, J (eds). The development of multiplicative reasoning in the learning of mathematics. New York: State university of New York press. 